\title{
Document Network and Conceptual and Social Structures of Clinical Endoscopy from 2015 to July 2021 Based on the Web of Science Core Collection: A Bibliometric Study
}

\author{
Sun Huh \\ Department of Parasitology and Institute of Medical Education, College of Medicine, Hallym University, Chuncheon, Korea
}

Background/Aims: The present study investigated the relevance and network of institutions, keywords, and authors' countries of the articles in Clinical Endoscopy published from 2015 to May 2021 based on the Web of Science Core Collection.

Methods: The Web of Science Core Collection was searched with the term Clinical Endoscopy as the publication title on July 12, 2021. All 776 citations published from 2015 to May 2021 and 2,964 articles citing those 776 articles were analyzed using Biblioshiny. Results: The corresponding authors were from 73 countries. Document coupling showed that the colorectal cancer-colonoscopyrandomized controlled trial cluster had the most significant impact and highest centrality. There were 442 articles with corresponding authors from Korea (57.0\%). The number of collaborative works by Korean authors with the authors of other countries was 33 (7.5\%). The articles were cited 2,964 times by corresponding authors from 37 countries.

Conclusions: The above results show that Clinical Endoscopy has published several studies on gastrointestinal endoscopy. A large proportion of citations $(84.7 \%$ ) were from outside Korea, indicating that the journal content is useful for global physicians. Collaborative work between authors from Korea and other countries should be encouraged to promote the journal. Clin Endosc 2021;54:641-650

Key Words: Bibliometrics; Colonoscopy; Colorectal neoplasms; Physicians; Republic of Korea

\section{INTRODUCTION}

Clinical Endoscopy (Clin Endosc, CE) is the official journal of the Korean Society of Gastrointestinal Endoscopy. This year, the International Digestive Endoscopy Network, Vietnam Association of Gastroenterology, Vietnam Federation for Digestive Endoscopy, and Thai Association for Gastrointestinal Endoscopy began to publish the journal, making it a co-official journal. The Korean Journal of Gastrointestinal En-

\footnotetext{
Received: July 20, $2021 \quad$ Revised: August 15, 2021

Accepted: August 17, 2021

Correspondence: Sun Huh

Department of Parasitology and Institute of Medical Education, College of Medicine, Hallym University, Hallimdaehak-gil 1, Chuncheon 24252, Korea Tel: +82-33-248-2652, Fax: +82-33-241-1672, E-mail: shuh@hallym.ac.kr ORCID: https://orcid.org/0000-0002-8559-8640

(c) This is an Open Access article distributed under the terms of the Creative Commons Attribution Non-Commercial License (http://creativecommons.org/ licenses/by-nc/3.0) which permits unrestricted non-commercial use, distribution, and reproduction in any medium, provided the original work is properly cited.
}

doscopy was published in Korea from November 1981 to July 2011 by the Korean Society of Gastrointestinal Endoscopy. In September 2011, the journal title was changed to Clinical Endoscopy, and an English-language policy was adopted. The purpose of the title change was to upgrade Clinical Endoscopy to a globally top-tier journal. After the journal title change, Clinical Endoscopy has been listed in PubMed Central (PMC) and PubMed since 2012, Scopus since 2013, and the Emerging Sources Citation Index since 2016. Ten years have passed since the launch of the English-only journal with a new title. From September 2011 to July 2021, 1,207 articles were published in Clinical Endoscopy. Its scope includes endoscopic diagnosis and interventions in the gastrointestinal and pancreaticobiliary tracts. No bibliometric analysis has yet investigated authors' affiliations, keywords, contents, and references for this journal. Therefore, the journal's network must be analyzed to explore its conceptual and social structures and to determine whether the journal's scope is well presented.

Previous bibliometric studies incorporating network anal- 
ysis have been conducted using articles from a single journal. For example, a prior study analyzed 50 years' worth of articles from the International Endodontic Journal, and bibliographic coupling highlighted themes, such as "endodontics", "root canal treatment" and "calcium hydroxide". ${ }^{1}$ Fifty years of articles in the Journal of Prosthetic Dentistry were analyzed in terms of categories, including keywords, cited documents, the countries and organizations of the authors, references, and sources cited. $^{2}$

Bibliometric analyses have frequently focused on specific subjects. Examples include articles on coronavirus disease 2019 (COVID-19) by Korean authors with the goal of analyzing the network of institutions, source journals, and keywords; ${ }^{3}$ analysis of journals, authors, and topics related to COVID-19 and Islamic finance listed in the Dimensions database; ${ }^{4}$ COVID-19 research published in nursing journals; ${ }^{5}$ historical diagnostic and therapeutic changes in ischemic stroke; ${ }^{6}$ scientific collaboration in North Korea; ${ }^{7}$ and physics papers from North Korea. ${ }^{8}$ Network analysis of a single journal may provide information about the impact of the journal in its scope area and scholarly journal network, trends in the keywords of articles, the network of journals, and the network of researchers by country. These analytical methods are widely used, but it is worthwhile for each journal to review its status.

This study explored the relevance and network of institutions, keywords, and authors' countries of articles published in Clinical Endoscopy based on a search of the Web of Science Core Collection on July 12, 2021. The articles citing Clinical Endoscopy were also analyzed separately. Furthermore, the article topics were clustered to identify research trends. The specific goals were as follows: first, to identify collaborative relationships among institutions; second, to characterize collaborative relationships among authors' countries; third, to determine clusters of institutions that published numerous articles; and fourth, to identify the main topics and clusters of research areas. For articles citing Clinical Endoscopy, the most relevant source, most relevant affiliation, and corresponding author's country were analyzed.

\section{MATERIALS AND METHODS}

\section{Ethics statement}

This was not a human-subject study; therefore, neither approval by the institutional review board nor obtaining informed consent was required.

\section{Study design}

This was a bibliometric network study of a journal using metadata from a literature database (the Web of Science Core
Collection). It was described in accordance with the STROBE reporting guidelines. ${ }^{9}$

\section{Setting/Variables}

The literature search was conducted on July 12,2021 . The Web of Science Core Collection was searched using the term Clinical Endoscopy as the publication title. All 776 articles were downloaded with information for all fields, including the author, affiliation, title, source, language, document type, keywords, Keywords Plus ${ }^{\circledast}$ (Clarivate; London, UK), abstract, and references. Furthermore, the articles citing the 776 articles of Clinical Endoscopy were also downloaded, of which the number was 2,964. Both of these data sources were used for the bibliometric analysis.

No variables were investigated.

\section{Data sources/Measurement}

Data acquired from the Web of Science Core Collection, as mentioned above, were used. Biblioshiny, an app version tool of Bibliometrix, ${ }^{10}$ was used for the analysis.

The following methods were used for data analysis. First, the main information was obtained from the Dataset menu; second, the most relevant affiliation was obtained from the Authors' menu; third, the corresponding author's country was obtained from the Authors' menu; fourth, the most frequent words and a word cloud were generated from the Document menu for Keywords Plus; fifth clustering by coupling was performed in the Clustering menu, where the unit of analysis was the document, coupling was measured by the author's keywords, impact was measured by the global citation score, and cluster labeling was performed using Keywords Plus; sixth, thematic evolution was analyzed using the Conceptual Structure menu, where the fields used Keywords Plus, the inclusion index was weighted by word occurrence, and the cut-off year was 2018; seventh, factorial analysis was conducted from the Conceptual Structure menu and; eighth, the country collaboration world map was generated from the Social Structure menu.

The most relevant sources and corresponding author's countries were evaluated for the articles citing Clinical Endoscopy.

No bias was reported during the database search and target article selection.

\section{Sample size/Statistical methods}

Since this was a study of the entire target literature, no study size estimation or post-hoc analysis was required.

Descriptive statistics were used. There were no inferential statistics. 


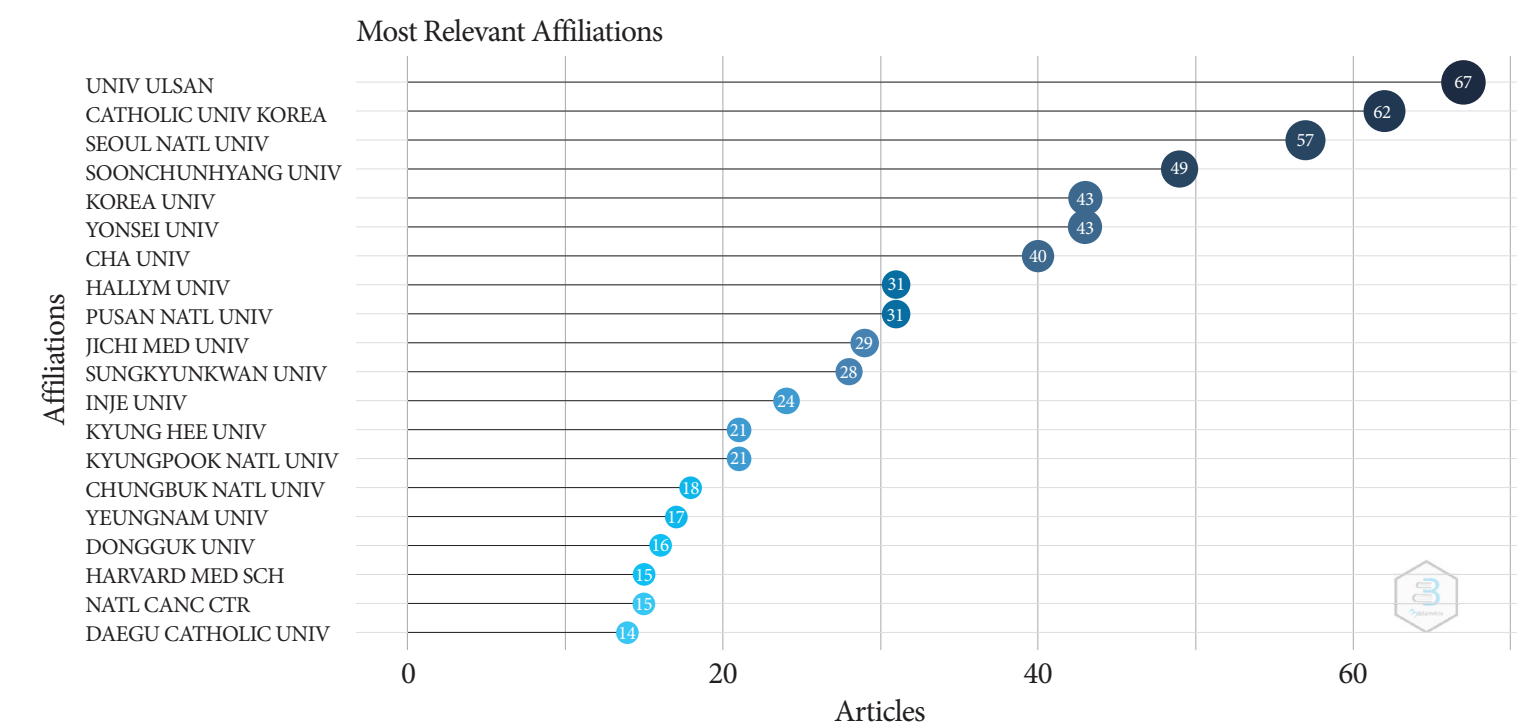

Fig. 1. Top 20 most relevant affiliations of the authors of Clinical Endoscopy articles in the Web of Science Core Collection generated from the Authors menu of Biblioshiny on July 12, 2021.

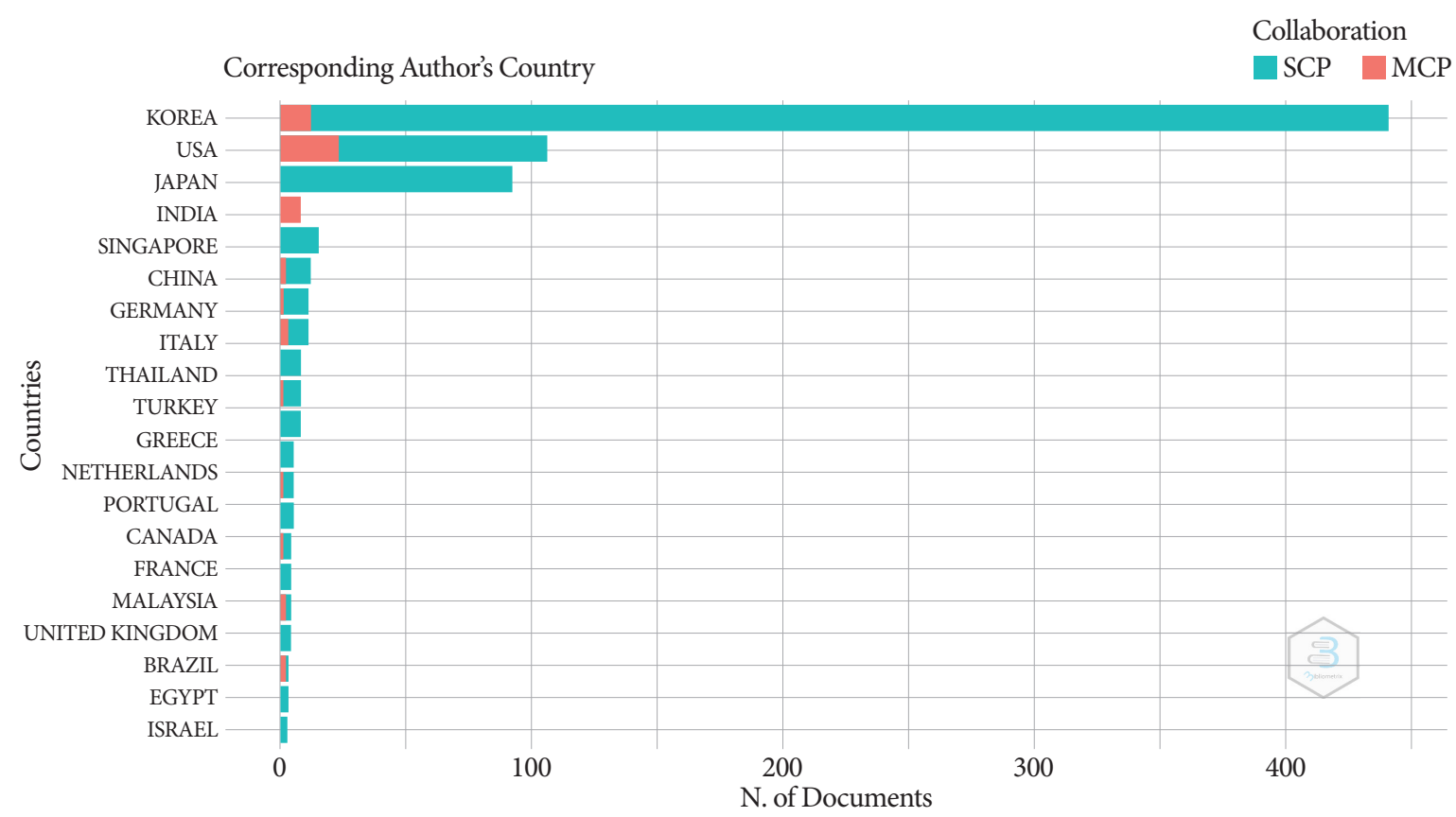

Fig. 2. Countries of the top 20 most relevant corresponding authors' of the articles of Clinical Endoscopy in the Web of Science Core Collection generated from the Authors menu of Biblioshiny on July 12, 2021. SCP, Single Country Publications; MCP, Multiple Country Publications. 


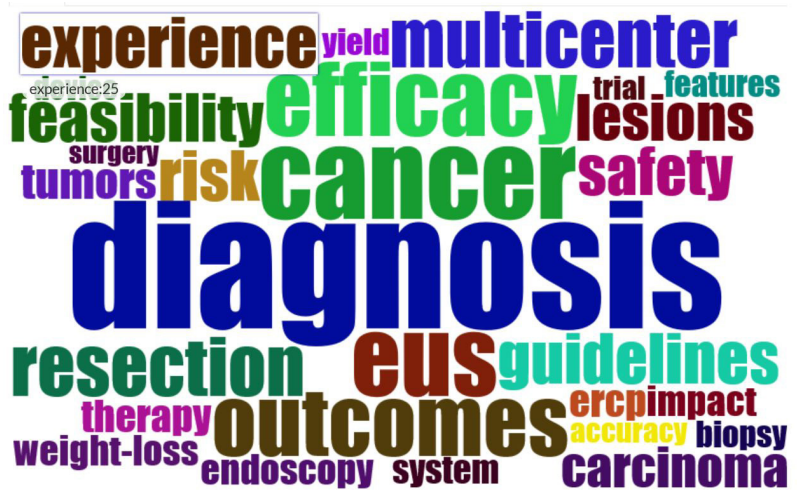

Fig. 3. Word cloud based on the most frequent words (Keywords Plus) used in the articles of Clinical Endoscopy in the Web of Science Core Collection generated from the Authors menu of Biblioshiny on July 12, 2021.

\section{RESULTS}

\section{Main information of the $\mathbf{7 7 6}$ articles published in Clinical Endoscopy}

The main information is tabulated in Supplementary Material 1 . The average number of citations per document was 4.39. There were 132 single-authored documents (17.0\%). The average number of authors per document was 4.55 (3,529/776).

The top 20 affiliations (out of 498 in total) are presented in Fig. 1 (Supplementary Material 2). The University of Ulsan, Catholic University of Korea, Seoul National University, Soonchunhayng University, and Korea University were the top five institutions in terms of the number of contributions.

Most relevant authors' countries: Top 20 corresponding authors' countries (out of 73 in total) are presented in Fig. 2 (Supplementary Material 3). Korea (442), the United States of America (USA) (106), Japan (92), India (15), and Singapore (12) were the top five countries.

\section{Most frequent words and the word cloud}

The most frequent words are listed in Supplementary Material 4. The word cloud from this list is shown in Fig. 3. Management, diagnosis, cancer, endoscopic ultrasonography (EUS), and efficacy were the top five most frequent words.

\section{Clustering and coupling of documents}

Given 250 units, a minimum cluster frequency per 1,000 units of 10, three labels per cluster, and a label size of 0.3 , the coupling map formed five clusters of documents identified by authors' keywords with the impact measure of global citation score and labeling by Keywords Plus (Fig. 4, Supplementary Material 5). The colorectal cancer-colonoscopy-randomized controlled trial cluster had the greatest impact and highest centrality.

\section{Thematic evolution and factorial network for} Keywords Plus as measures of conceptual structure

The thematic evolution of Keywords Plus is presented in Fig. 5 (Supplementary Material 6). Recent studies have shifted toward management, outcomes, and long-term outcomes determined using EUS; risk factors; and diagnosis. The factorial network showed two clusters when multiple correspondence analysis was performed for the Keywords Plus field, with a minimum number of terms of 50 , an automatically calculated cluster number, a label size of 10 , and a minimum number of documents of five for graphic parameters (Fig. 6, Supplementary Material 7). One was a cluster of endoscopy-related keywords, and the other was a cluster encompassing mucosal resection, early gastric cancer, and clinical outcomes.

\section{Collaborative world map as a measure of social structure}

There were 1,100 collaborations between authors from different countries. Because there are artcles from multiple countries, the number of collaborations could exceed the number of articles. (Supplementary Material 8). The world map (Fig. 7) showed that the most frequent collaborations were between Korea and the USA (18), followed by those between Korea and Japan (4), and between the USA and Brazil (4). Thirty-three of the 442 Korean-authored articles involved international collaboration $(7.5 \%)$. The frequency of international collaboration for articles with American authors was 33 out of 106 articles (31.1\%).

\section{Most relevant source of 2,964 citing articles}

The top 20 source journal titles (out of 775 in total) are presented in Fig. 8 (Supplementary Material 9). The top five most frequently cited journals were Clinical Endoscopy (259), Endoscopy (95), Gastrointestinal Endoscopy (90), Surgical Endoscopy and Other Interventional Techniques (76), and Endoscopy International Open (74).

\section{Most relevant corresponding authors' countries}

The top 20 countries (out of 37 in total) are presented in Fig. 9 (Supplementary Material 10). The USA (607), China (457), Korea (456), Japan (332), and Italy (152) were the most frequently cited countries. 


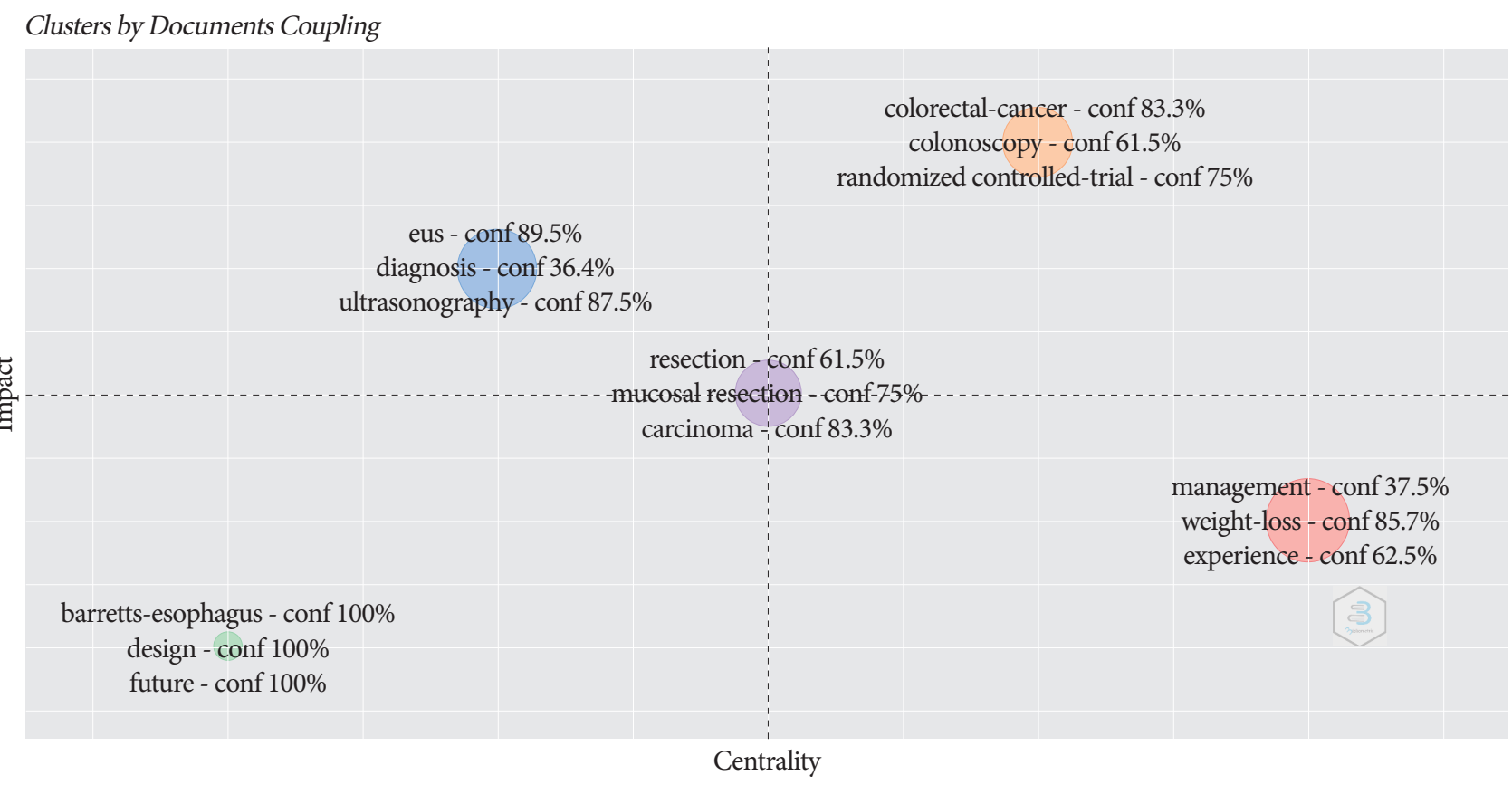

Fig. 4. Cluster coupling measured by authors' keywords with the impact measure of global citation score and cluster labeling by Keywords Plus in Clinical Endoscopy articles in the Web of Science Core Collection generated from the Clustering menu of Biblioshiny on July 12, 2021. Conf, confidence level; EUS, endoscopic ultrasound.

\section{DISCUSSION}

The authors of Clinical Endoscopy were from 498 institutions worldwide. The corresponding authors were from 73 countries. Document coupling showed that the colorectal cancer-colonoscopy-randomized controlled trial cluster had the most significant impact and highest centrality. There were two clusters of Keywords Plus; one comprised endoscopy-related keywords, while the other was a cluster of mucosal resection-early gastric cancer-clinical outcomes. Out of 776 articles, there were 1,100 collaborations between authors from different countries. There were 442 articles with corresponding authors from Korea. The number of collaborative works between Korean authors and authors from other countries was 33 (7.5\%). The most frequent collaborations (18) were found between the authors from Korea and those from the USA. Clinical Endoscopy has been cited 2,964 times in articles published in 775 journals, with corresponding authors from 37 countries.

Of the 776 articles, 443 (57.0\%) were from authors in Korea. The top nine authors' affiliations were institutions in Korea (Supplementary Material 2). This finding is not surprising because the journal is published by an academic society in Korea, and the main clients of the journal have been society members. In 2021, Clinical Endoscopy became the co-official journal of the International Digestive Endoscopy Network,
Vietnam Association of Gastroenterology, Vietnam Federation for Digestive Endoscopy, and Thai Association for Gastrointestinal Endoscopy. Therefore, the number of submissions from members of these societies is anticipated to increase over time. These changes will enhance the regional diversity of the journal. The effort to invite other societies in the same field is a timely policy to broaden the diversity of authors and affiliations.

As stated by Clarivate Analytics, "the data in Keywords Plus are words or phrases that frequently appear in the titles of an article's references but do not appear in the title of the article itself. Based upon a special algorithm that is unique to Clarivate Analytics databases, Keywords Plus enhances the power of cited-reference searching by searching across disciplines for all the articles that have cited references in common". ${ }^{11}$ Since Keywords Plus represents a source of machine-generated terms through cited references, it was selected for the network analysis of topics to emphasize the impact of the cited references. Although "management" was the most frequently used word, "diagnosis", "cancer" and "EUS" were central words in the word cloud (Fig 3, Supplementary Material 4). Therefore, the words "diagnosis", "cancer" and "EUS" were most frequently related to other words in the Keywords Plus network.

The coupling map of documents measured by authors' keywords showed that the colorectal cancer-colonoscopy-ran- 


\section{C cLincal endoscopy}

2015-2018

2019-2021

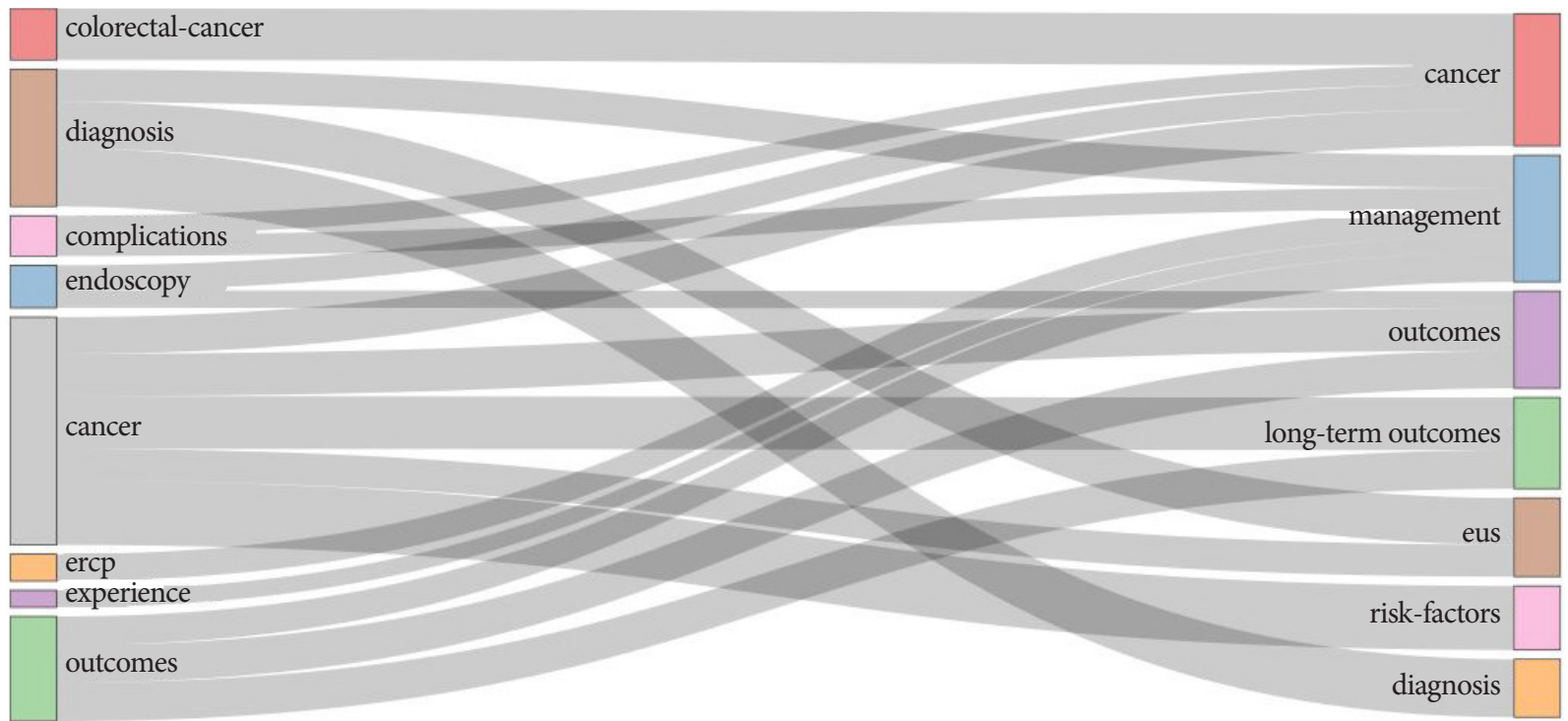

Fig. 5. Thematic evolution (Keywords Plus) of the articles of Clinical Endoscopy in the Web of Science Core Collection generated from the Conceptual structure menu of Biblioshiny on July 12, 2021. ERCP, endoscopic retrograde cholangiopancreatography; EUS, endoscopic ultrasound.

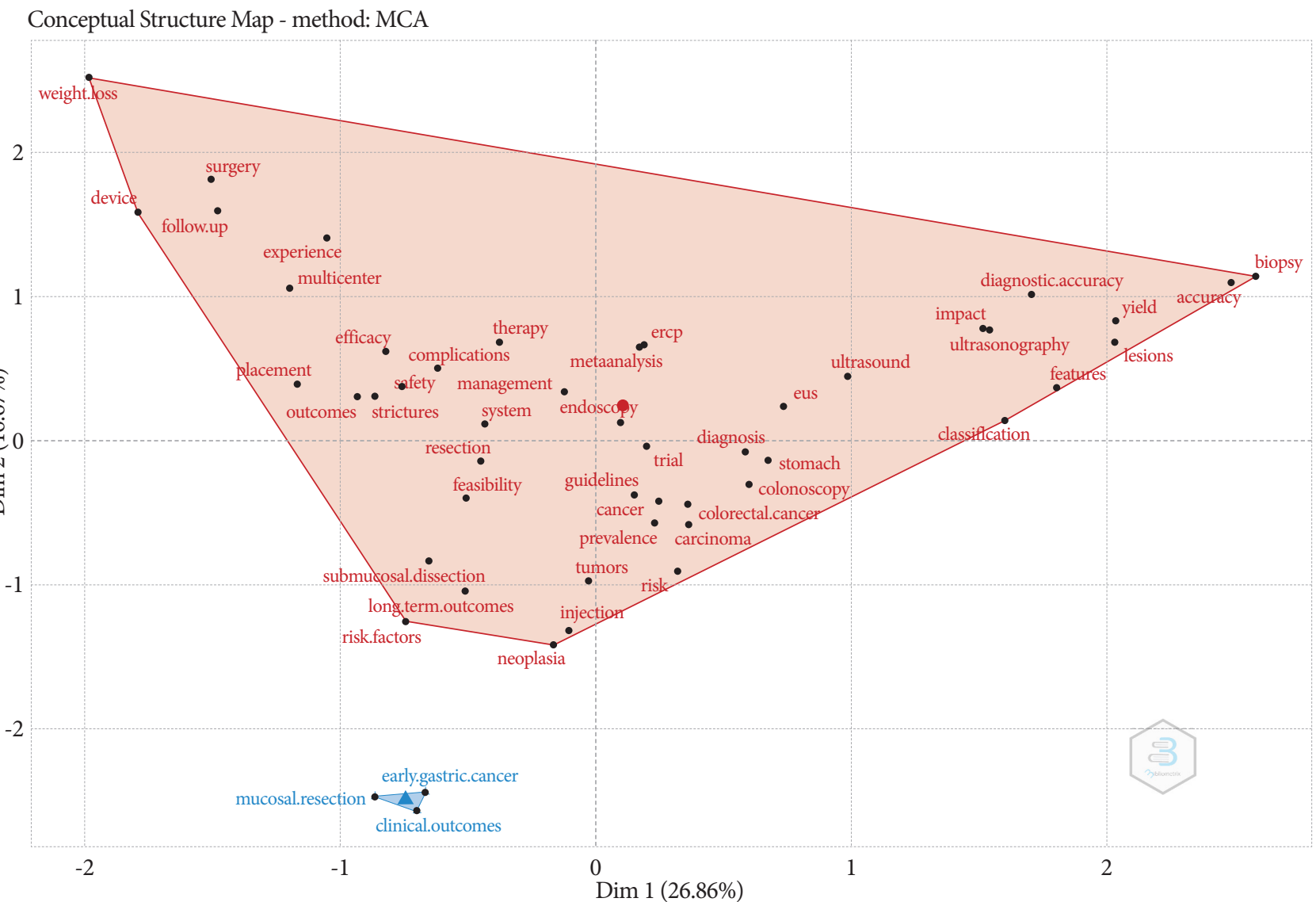

Fig. 6. Conceptual structure map (Keywords Plus) based on the factorial analysis of the articles of Clinical Endoscopy in the Web of Science Core Collection generated from the Conceptual structure menu of Biblioshiny on July 12, 2021. ERCP, endoscopic retrograde cholangiopancreatography; EUS, endoscopic ultrasound; MCA, multiple corresponding analysis; Dim1, Dimension; and Dim2, Dimension 2. 
Country Collaboration Map

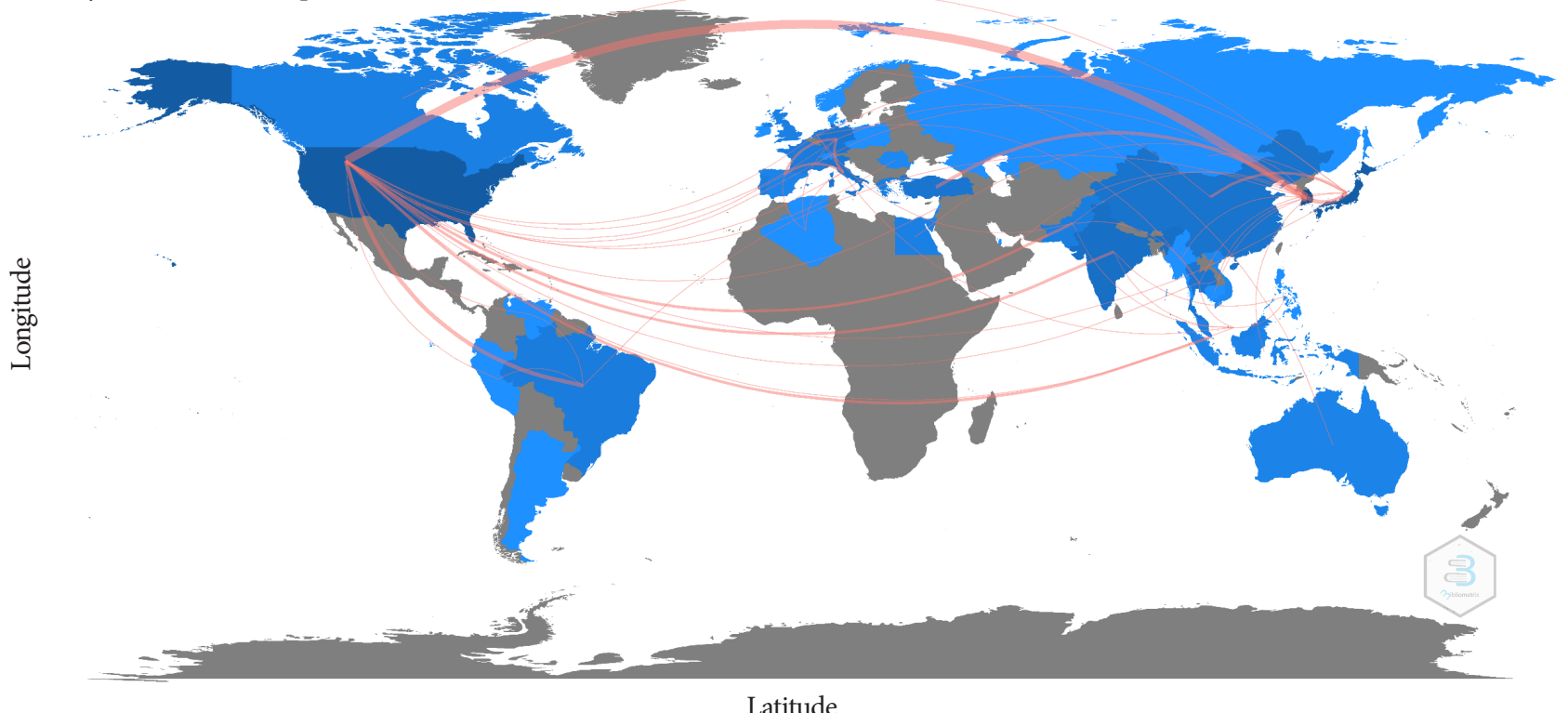

Fig. 7. Country collaboration map of Korean authors of the articles of Clinical Endoscopy in the Web of Science Core Collection generated from the Social Structure menu of Biblioshiny on July 12, 2021.

\section{Most Relevant Sources}

CLINICAL ENDOSCOPY

\section{ENDOSCOPY}

GASTROINTESTINAL ENDOSCOPY

SURGICAL ENDOSCOPY AND OTHER INTERVENTIONAL TECHNI ENDOSCOPY INTERNATIONAL OPEN

DIGESTIVE ENDOSCOPY

WORLD JOURNAL OF GASTROENTEROLOGY

DIGESTIVE DISEASES AND SCIENCES

MEDICINE

ङ ENDOSCOPIC ULTRASOUND

ENDOSCOPIC ULTRASOUND

↔ JOURNAL OF GASTROENTEROGY AND HEPATOLOGY GUT AND LIVER

BMC GASTROENTEROLOGY

REVISTA ESPANOLA DE ENFERMEDADES DIGESTIVAS OBESITY SURGERY

CUREUS

ACG CASE REPORTS JOURNAL

SCANDINAVIAN JOURNAL OF GASTROENTEROLOGY WORLD JOURNAL OF GASTROINTESTINAL ENDOSCOPY

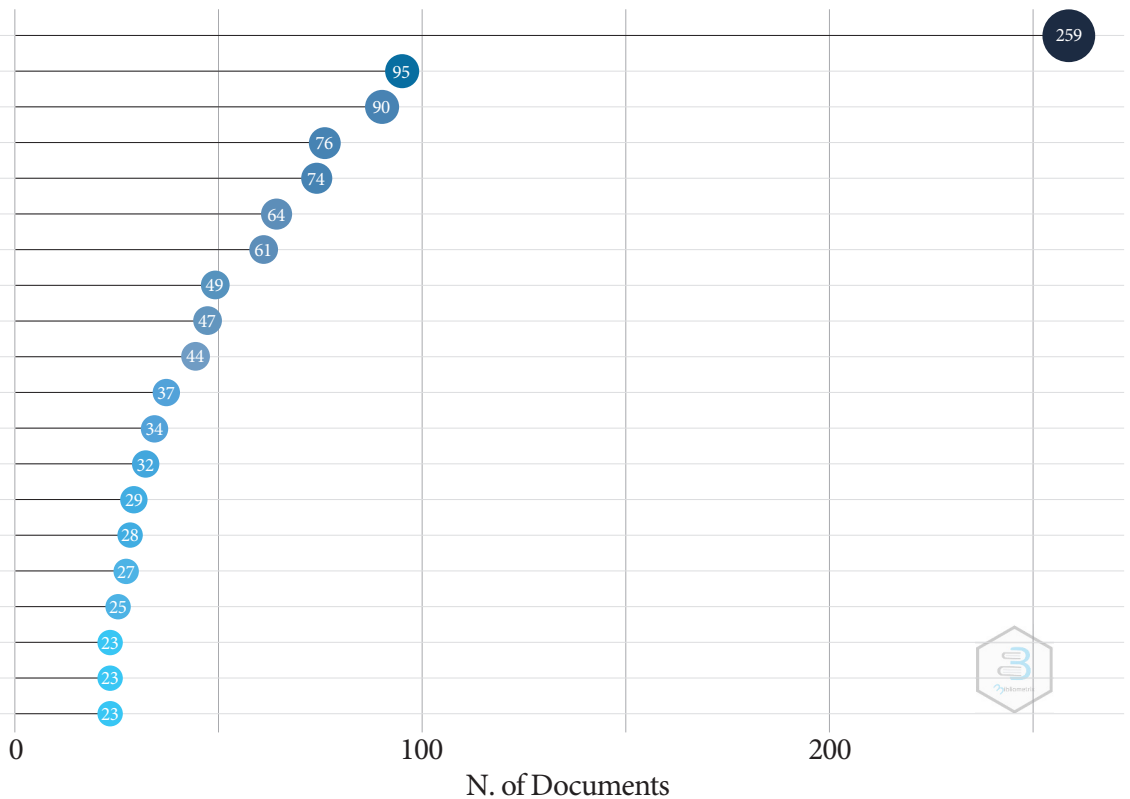

Fig. 8. Top 20 most relevant journal titles of articles citing Clinical Endoscopy in the Web of Science Core Collection generated from the Source menu of Biblioshiny on July 12, 2021. 


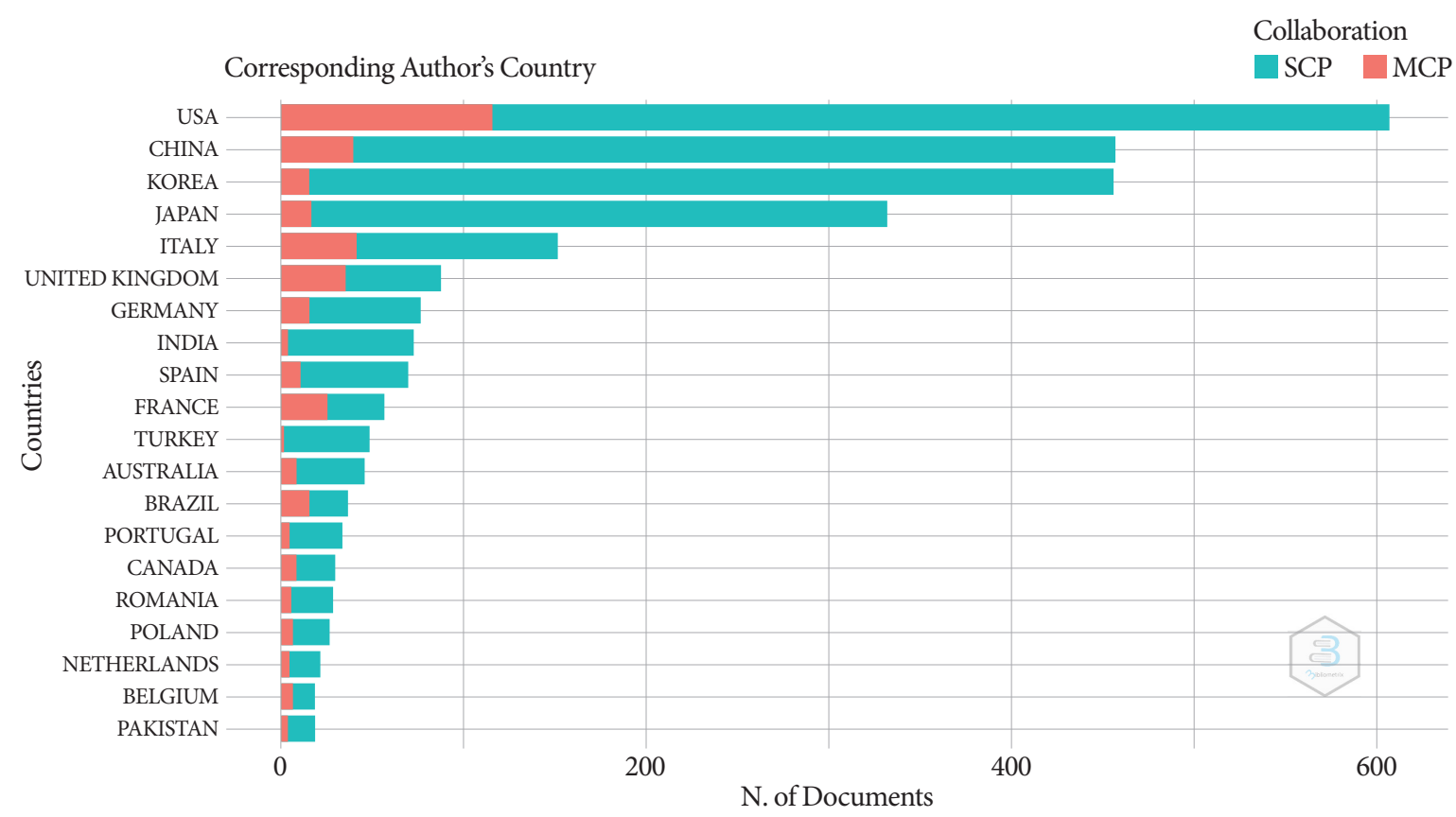

Fig. 9. Corresponding author's country. SCP, Single Country Publications; MCP, Multiple Country Publications.

domized controlled trial cluster had the most significant impact and centrality relative to the other four clusters (Fig. 4, Supplementary Material 5). This finding is in contrast with the keyword plus network presented in the word cloud, which can be explained by the fact that word clouds present relationships among single words. Three authors' keywords (colorectal cancer-colonoscopy-randomized controlled trial) concurrently appeared in journal documents, so that these three words formed a cluster reflecting the most influential keywords in the journal.

Thematic evolution (Fig. 5) showed the changes in the main Keywords Plus. Patient management and outcomes were the topics that rapidly evolved. This evolution may change according to the changes in the topics. The factorial network of Keywords Plus showed no significant differences (Fig. 6). Multiple correspondence analysis was used for dimensionality reduction, which is a "data analysis technique for nominal categorical data, used to detect and represent underlying structures in a dataset. It does this by representing data as points in a low-dimensional Euclidean space". ${ }^{12}$ A small portion of the mucosal resection-early gastric cancer-clinical outcomes cluster existed; however, its proportion was negligible. Therefore, the conceptual structure of Keywords Plus based on the factorial analysis indicated that the journal's content focused primarily on endoscopy-related issues.
Collaborative work with researchers in other countries has been generally encouraged to generate more robust research outcomes in terms of regional diversity, increasing the applicability of the results to patient care. The collaboration rate of Korean authors was $7.5 \%$, while that of American authors was 31.1\% (Fig. 7, Supplementary Material 8). An international network for research should be encouraged for Korean authors. Society funding for members to engage in international collaborative research may be an incentive to improve international collaboration. Most Korean authors' collaborate with American researchers (54.5\%), and they should broaden their range of collaboration. This process may also be accelerated by invitations extended to other countries' societies to become partners in journal publishing from 2021.

It is promising from the standpoint of journal promotion to see the citation frequency, citing source titles, and citing authors' countries. The citation frequency, 2,964, was 4.39 times the number of articles, reflecting outstanding performance. The number of citations of journal titles was 775 (Fig. 8, Supplementary Material 9). This is also an excellent result. These results were made possible by the high quality of the articles and the indexing of the journal in major international databases, including PubMed, PubMed Central, Scopus, and Web of Science Core Collection. Indexing in international databases provides a higher chance of articles being accessed by 
researchers. $^{13}$

The journal literature was limited to studies indexed in the Web of Science Core Collection. The number of articles in Clinical Endoscopy from 2011 to July 2021 was 1,211. An analysis of studies published before 2015 was not conducted. However, if articles before 2015 had been included, the findings might have been different. The present study only included articles from the most recent 6 years.

Because the subject of analysis was limited to a single journal, it was not possible to generalize the present results. However, the top three most frequent countries of citing authorsthe USA, China, and Korea-might reflect a common pattern for many medical journals published by academic societies in Korea because the USA and China are currently the two most productive countries in scientific publishing.

What actions will the journal take to improve its bibliometric properties, including citation frequency? First, Clinical Endoscopy announced a preprint policy in 2021 that allowed the submission of preprints. This is a timely policy for journal promotions. Only 28 out of 383 Asian academic society journals registered in the Science Citation Index Expanded in 2020 announced preprint policies. ${ }^{14}$ However, half of the editors in Korea do not agree with the need for preprints because of concerns regarding the lack of scientific integrity, stealing ideas/ scooping data, priority issues regarding research ideas, and copyright problems. ${ }^{15}$ Nonetheless, preprints are a rising trend in journal publishing in the natural sciences, social sciences, and humanities. ${ }^{16}$

Clinical Endoscopy still has not yet announced a data-sharing policy. Data constitute an essential topic of journal publishing, as even data journals have been published. ${ }^{17}$ Ten out of 133 Asia Science Citation Index Expanded (SCIE) journals in 2019 had announced data sharing policies (7.5\%), ${ }^{18}$ while 12 of 59 Korean academic society-published SCIE journals had done so in 2020. ${ }^{19}$ Clinical Endoscopy needs to consider announcing a data-sharing policy to guarantee the reproducibility of analyses. Verification of clinical data sharing policies has been recommended by the International Committee of Medical Journal Editors, according to which the authors can make decisions about clinical data sharing. It is unclear whether a data sharing policy is helpful in increasing the number of submissions or the citation frequency. Furthermore, there are concerns regarding the decreased number of submissions. However, this policy will increase the transparency of the journal.

This analysis of the relevance and network of institutions, keywords, and authors' countries of the articles in Clinical Endoscopy based on the Web of Science Core Collection showed that the journal has produced articles on endoscopy and has been cited by 775 journals, including leading endoscopy-relat- ed journals. Although the proportion of Korean authors was $57.5 \%$ (442/776), only $15.3 \%(456 / 2,964)$ of the citations were from Korea. Therefore, it can be stated that the journal content is helpful for global physicians and researchers. Collaborative work between authors from Korea and other countries needs to be encouraged to further promote the journal.

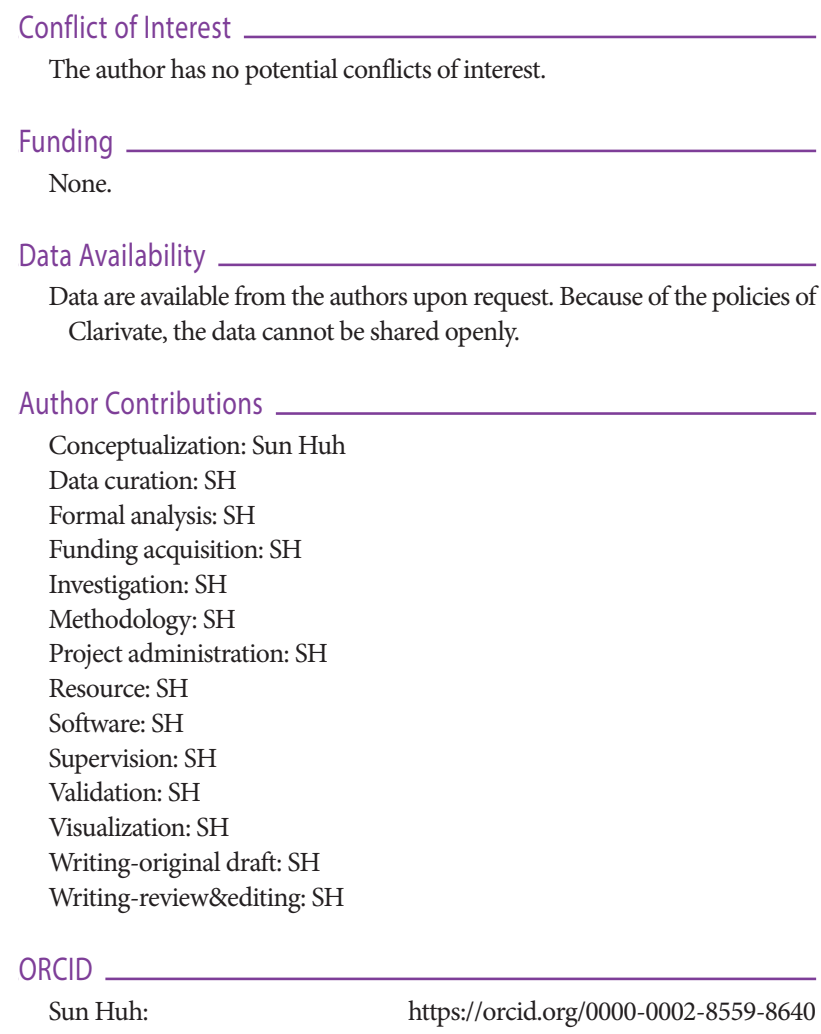

\section{REFERENCES}

1. Khan AS, Rehman SU, Ahmad S, AlMaimouni YK, Alzamil MAS, Dummer PMH. Five decades of the International Endodontic Journal: bibliometric overview 1967-2020. Int Endod J 2021;54:1819-1839.

2. Alhajj MN, Al-Sanabani FA, Alkheraif AA, Simran A, Alqerban A, Samran A. Bibliometric analysis and evaluation of the Journal of Prosthetic Dentistry from 1970 to 2019. J Prosthet Dent 2021:S00223913(21)00279-1.

3. Kim KW, Jeong GH. Network of institutions, source journals, and keywords on COVID-19 by Korean authors based on the Web of Science Core Collection in January 2021. Sci Ed 2021;8:47-56.

4. Rusydiana AS. Bibliometric analysis of journals, authors, and topics related to COVID-19 and Islamic finance listed in the Dimensions database by Biblioshiny. Sci Ed 2021;8:72-78.

5. Oh J, Kim A. A bibliometric analysis of COVID-19 research published in nursing journals. Sci Ed 2020;7:118-124.

6. Kim Y, Yoon DY, Kim JE, Lee JH, Song HK, Bae JS. Historical diagnostic and therapeutic changes of ischemic stroke based on the highly cited articles. Sci Ed 2020;7:156-162.

7. Choi HW, Choi YJ, Kim S. Network analysis of scientific collaboration in North Korea. Sci Ed 2019;6:25-34. 
8. Kim K, Chung Y. Bibliographic and content analysis of physics papers from North Korea indexed in the Scopus from 2005 to 2018. Sci Ed 2019;6:35-40.

9. Vandenbroucke JP, von Elm E, Altman DG, et al. Strengthening the Reporting of Observational Studies in Epidemiology (STROBE): explanation and elaboration. Ann Intern Med 2007;147:W163- W194.

10. Aria M, Cuccurullo C. Bibliometrix: An R-tool for comprehensive science mapping analysis. J Informetr 2017; 11: 959-975.

11. Clarivate Analytics. KeyWord Plus generation, creation, and changes [Internet]. London; Clarivate Analytics; c2018 [cited 2021 July 10]. Available from: https://support.clarivate.com/ScientificandAcademicResearch/s/article/KeyWords-Plus-generation-creation-and-changes?language $=$ en_US

12. Wikipedia. Multiple correspondence analysis [Internet]. San Francisco, CA: Wikimedia Foundation; c2020 [cited 2021 July 10]. Available from: https://en.wikipedia.org/wiki/Multiple_correspondence_analysis.

13. Jeong $\mathrm{GH}$, Huh S. Increase in frequency of citation by SCIE journals of
non-Medline journals after listing in an open access full-text database. Sci Ed 2014;1:24-26.

14. Choi YJ, Choi HW, Kim S. Preprint acceptance policies of Asian academic society journals in 2020. Sci Ed 2021;8:10-17.

15. Yi HJ, Huh S. Korean editors' and researchers' experiences with preprints and attitudes towards preprint policies. Sci Ed 2021;8:4-9.

16. Vuong QH. The rise of preprints and their value in social sciences and humanities. Sci Ed 2020;7:70-72.

17. Kim J. An analysis of data paper templates and guidelines: types of contextual information described by data journals. Sci Ed 2020;7:16-23.

18. Choi YJ, Choi HW, Kim S. Compliance of "Principles of transparency and best practice in scholarly publishing" in Korean academic society-published journals listed in Journal Citation Reports. Sci Ed 2020;7:24-33.

19. Choi HW, Choi YJ, Kim S. Compliance of "Principles of transparency and best practice in scholarly publishing" in academic society published journals. Sci Ed 2019;6:112-121. 